\title{
Intussuscepted Meckel's diverticulum within its own lumen
}

\author{
Patoulias Dimitrios ${ }^{1}$, Chatzopoulos Kyriakos², Patoulias Ioannis ${ }^{3}$
}

${ }^{1}$ Department of Internal Medicine, General Hospital of Veria, Veria, Greece, ${ }^{2}$ Department of Pathology, General Hospital G. Gennimatas, Thessaloniki, Greece, ${ }^{3} 1^{\text {st }}$ Department of Pediatric Surgery, Aristotle University of Thessaloniki, General Hospital G. Gennimatas, Thessaloniki, Greece
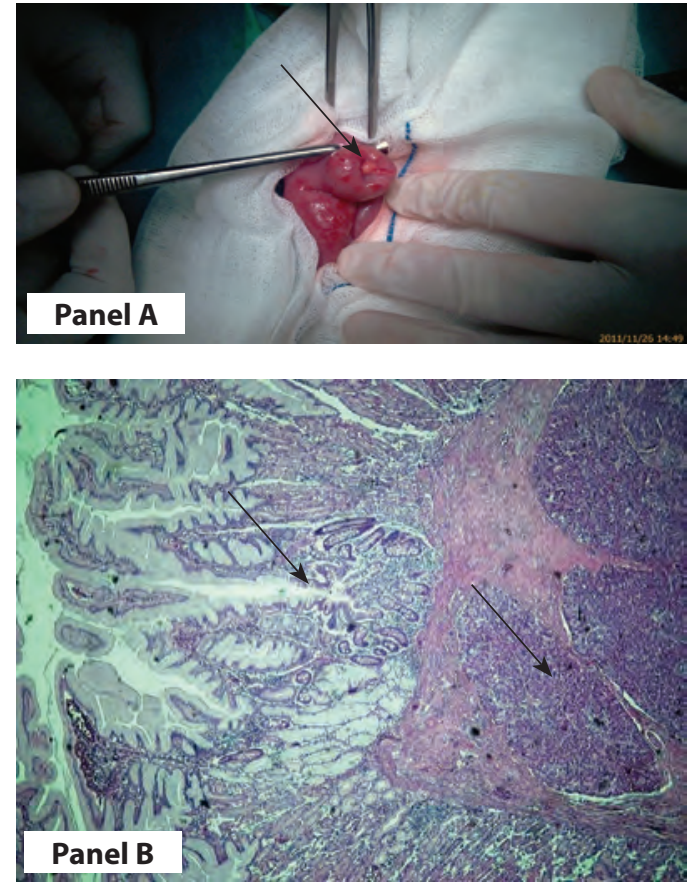

A 8-year old boy with negative medical history presented to the Emergency Department (ED) complaining of colicky, abdominal pain over two days. This pain progressed to being generalized in nature, while a bloody, mucoid stool was noted prior to ED presentation. Physical examination revealed tenderness and mild rigidity in the right abdominal wall. Laboratory tests were in- dicative of inflammation. Abdominal x-ray findings were unremarkable. Performance of ultrasonography excluded the presence of pathology of the intra-abdominal solid organs, the presence of free fluid within the peritoneal cavity, or enlarged intra-abdominal lymph nodes, whereas the typical target (or doughnut) and pseudokidney signs for intussusception were absent.

The patient underwent urgent exploratory laparotomy, after a Lanz incision. The appendix was not inflamed, but preventive appendectomy was performed. Further, a thorough investigation of the peritoneal cavity followed. Seventy centimeters from the ileocecal valve, a Meckel's diverticulum was found intussuscepted within its own lumen (Panel A).

Intraoperatively we observed the hemorrhagic infiltration of its wall, along with the presence of ectopic tissue (Panel A, black arrow). We then performed excision of the Meckel's diverticulum. The postoperative course was uneventful and the patient was discharged home in good general condition on the second postoperative day. Histological examination (Panel B, Hematoxylin eosin stain, X40) revealed that the Meckel's diverticulum was lined with gastric and in- 
testinal epithelium (red arrow), while inside the muscular layer, islands of ectopic pancreatic tissue were observed (black arrow). The wide base or the small dimensions of the diverticulum have been reported as precipitating factors for the development of intussusception of Meckel's diverticulum within its lumen, but these were not observed in the present case $(1,2)$. Histological findings indicative for the presence of an active ulcer at the luminal orifice on the antimesenteric bowel wall could constitute a predisposing factor for intussuscepted Meckel's diverticu$\operatorname{lum}(1,3)$.

Key words: Meckel's diverticulum - Intussusception Lumen • Inflammation.

Authors' contributions: Conception and design: DP and IP; Acquisition, analysis and interpretation of data: DP and IP; Drafting the article: DP, KC and IP; Revising it critically for important intellectual content: DP and IP; Approved final version of the manuscript: DP, KC and IP.
Conflict of interest: The authors declare that they have no conflict of interest.

Correspondence:

dipatoulias@gmail.com

Tel.: + 302310 225083; Fax.: + 302310225083

Received: 7 September 2017; Accepted: 13 November 2017

\section{References}

1. Kassir R, Debs T, Boutet C, Baccot S, Abboud K, Dubois J, et al.Intussusception of the Meckel's diverticulum within its own lumen: Unknown complication. Int J Surg Case Rep. 2015;10:111-4.

2. Codrich D, Taddio A, Schleef J, Ventura A, Marchetti F. Meckel's diverticulum masked by a long period of intermittent recurrent subocclusive episodes. World J Gastroenterol. 2009;15(22):280911 .

3. Chen JJ, Lee HC, Yeung CY, Chan WT, Jiang CB, Sheu JC, et al. Meckel's Diverticulum: Factors Associated with Clinical Manifestations. ISRN Gastroenterol. 2014;390869.doi: 10.1155/2014/390869 